\title{
COHERENT RINGS OF DIMENSION TWO
}

\author{
HO KUEN NG
}

\begin{abstract}
We first continue the investigation of the finitely presented dimension, especially in the local case, then we propose a scheme for classifying commutative rings. Commutative coherent rings of finitely presented dimension two and global dimension two will be studied.
\end{abstract}

1. Introduction. Let $R$ be a commutative ring with $1 \neq 0$ in this paper.

In [2], we introduced the finitely presented dimensions of modules and commutative rings. For $A$ an $R$-module, we defined the finitely presented dimension of $A$, f.p.dim $A$, as $\inf \left\{n \mid\right.$ there exists an exact sequence $P_{n+1} \rightarrow P_{n} \rightarrow \cdots \rightarrow P_{0} \rightarrow A \rightarrow$ 0 of $R$-modules, where each $P_{i}$ is projective, and $P_{n+1}, P_{n}$ are finitely generated $\}$. If no such exact sequence exists, we say that $A$ has infinite finitely presented dimension. We also defined the finitely presented dimension of the $\operatorname{ring} R$, f.p.dim $R$, as $\sup \{$ f.p.dim $A \mid A$ is a finitely generated $R$-module .

In [2], we showed that the finitely presented dimension has the properties that we expect of a 'dimension' if the ring in question is coherent. It was also proved that for a coherent $\operatorname{ring} R, \operatorname{gl} \cdot \operatorname{dim} R=\sup (w . g l \cdot \operatorname{dim} R$, f.p.dim $R-1$ ).

In $\S 2$ of this paper, we investigate the finitely presented dimension of a quasi-local ring, and obtain results concerning coherent rings of finitely presented dimension 2 . We then point out a scheme for classifying rings according to their global dimensions, weak global dimensions and finitely presented dimensions. In $\S 3$, we show that the classification of quasi-local rings of global dimension 2 in [3] can be conveniently described by our scheme, and we look at the spectrum of prime ideals of coherent rings of global dimension 2.

2. Finitely presented dimension of coherent quasi-local rings. We first make the following

Definition 2.1. Let $R$ be a ring. An $R$-module $A$ is said to be almost finitely presented if $A=B \oplus C$, where $B$ is finitely presented, and $C$ is nonfinitely generated free.

THEOREM 2.2. Let $R$ be a ring over which projective modules are free. Let $A$ be an $R$-module with f.p.dim $A=1$. Then $A$ is almost finitely presented.

Received by the editors September 22, 1983.

1980 Mathematics Subject Classification. Primary 13D05.

(c) 1984 American Mathematical Society $0002-9939 / 84 \$ 1.00+\$ .25$ per page 
Proof. Let $0 \rightarrow K \rightarrow F \rightarrow A \rightarrow 0$ be an exact sequence with $K$ finitely presented and $F$ projective, and hence free. We fix a finite set of generators for $K$, and a set of free generators for $F$. We express the generators of $K$ as unique linear combinations of the free generators of $F$. Let $f_{1}, \ldots, f_{n}$ be involved in these linear combinations. Let $a_{1}, \ldots, a_{n}$ be their images under the map $F \rightarrow A$, and let $B=\left(a_{1}, \ldots, a_{n}\right)$. Let $C$ be the submodule of $A$ generated by the images of the other free generators of $F$, say $f_{j} \mapsto a_{j}$. Clearly we have $A=B+C$.

If $r_{1} a_{1}+\cdots+r_{n} a_{n}=r_{n+1} a_{n+1}+\cdots+r_{n+s} a_{n+s}$, where the $r_{i}$ 's are in $R$, and $a_{n+1}, \ldots, a_{n+s}$ are among the generators of $C$ as described above, then $r_{1} f_{1}+\cdots+$ $r_{n} f_{n}-\left(r_{n+1} f_{n+1}+\cdots+r_{n+s} f_{n+s}\right)$ is in $K$, and hence is a linear combination of $f_{1}, \ldots, f_{n}$. By the freeness of $F, r_{n+1}=\cdots=r_{n+s}=0$, showing that $B \cap C=0$, and so $A=B \oplus C$.

The exact sequence $0 \rightarrow K \rightarrow\left(f_{1}, \ldots, f_{n}\right) \rightarrow B \rightarrow 0$ shows that $B$ is finitely presented. As f.p.dim $A=1, A$ is nonfinitely generated, and so $F$ is nonfinitely generated. $C$ is nonfinitely generated free because it is isomorphic to the submodule of $F$ generated by the free generators other than $f_{1}, \ldots, f_{n}$. This completes the proof.

Proposition 2.3. Let $A$ be an $R$-module.

(1) f.p.dim $A=0$ if and only if $A$ is finitely presented.

(2) If $R$ is quasi-local and f.p.dim $A=1$, then $A$ is almost finitely presented.

(3) If $R$ is coherent and $A$ is almost finitely presented, then f.p.dim $A=1$.

(4) If $R$ is quasi-local coherent, then f.p.dim $A=1$ if and only if $A$ is almost finitely presented.

Proof. (1) follows from definition. (2) follows from 2.2. (3) follows from the fact that a finitely generated submodule of a free module over a coherent ring is finitely presented. (4) is an immediate consequence of (2) and (3).

Proposition 2.4. A coherent quasi-local ring cannot have finitely presented dimension 2.

Proof. Else, there is an ideal $I$ with f.p.dim $I=1$, from [2]. Then $I=B \oplus C$, where $B$ is finitely presented, and $C$ is nonfinitely generated free. But $C$, being a free ideal of the ring, must be principal, a contradiction.

Among the consequences of 2.4 , we see that a non-Noetherian hereditary ring cannot be quasi-local. In other words, a quasi-local hereditary ring must be Noetherian. Also, it follows from 2.4 that no valuation rings can have finitely presented dimension 2, a fact proved in [2].

However, we remark that if $R$ is not quasi-local, f.p.dim $A=1$ need not imply that $A$ is almost finitely presented, because [2] showed that there is a coherent ring $R$ with f.p.dim $R=2$, and so there is an ideal with finitely presented dimension 1 .

Proposition 2.5. Let $R$ be non-Noetherian, and over which projective modules are free. Then f.p.dim $R \geqslant \operatorname{gl} . \operatorname{dim} R+1-\sup \{$ p.d. $A \mid A$ is a finitely presented $R$-module \}. 
Proof. It follows easily from the fact that over such a ring, a module with finitely presented dimension 1 is almost finitely presented, and so its projective dimension is the same as that of its finitely presented direct summand.

In [2], we saw that a ring is Noetherian if and only if it has finitely presented dimension 0 . We said that the finitely presented dimension is a measure of how far away a ring is from being Noetherian. The following results point further in this direction.

Proposition 2.6. If f.p.dim $R=2$, and $R_{p}$ is coherent for all prime ideals $p$ of $R$, then $R$ is non-Noetherian, all localizations of which with respect to prime ideals are Noetherian.

Proof. It follows from [2] that a ring cannot have finitely presented dimension 1.

COROLlary 2.7. A coherent ring with finitely presented dimension 2 is nonNoetherian, all localizations of which with respect to prime ideals are Noetherian.

For convenience, we call a ring $R$ an $(a, b, c)$-ring if w.gl.dim $R=a$, gl.dim $R=b$, and f.p.dim $R=c$.

Before we move on to the next set of results, we need the following

Proposition 2.8. Let $R$ be a coherent ring. Consider the following three conditions.

(1) w.gl.dim $R<$ gl.dim $R$.

(2) f.p.dim $R>\sup \left\{\right.$ f.p.dim $R_{m} \mid m$ is a maximal ideal $\}$.

(3) gl.dim $R>\sup \left\{\operatorname{gl} . \operatorname{dim} R_{m} \mid m\right.$ is a maximal ideal $\}$.

Assuming any one of these, the remaining two are equivalent.

Proof. This follows by applying Theorem 3.4 of [2], and distinguishing between the finite and infinite cases.

Coherent rings were defined as a generalization of Noetherian rings. However some important properties possessed by the latter are not shared by the former, notably the Hilbert Basis Theorem. If we define $\mathscr{C}$ to be the class of coherent rings with finitely presented dimension $\leqslant 2$, then $\mathscr{C}$ is much more similar to the class of Noetherian rings. Examples of rings of finitely presented dimension 2 are given in [2].

Proposition 2.9. Let $R \in \mathscr{C}$. Then gl.dim $R<\infty$ if and only if Krull dimension $R<\infty$ and $R_{p}$ is regular, for any prime ideal $p$ of $R$.

Proof. We need ony consider f.p.dim $R=2$. For necessity, we note that each localization of $R$ with respect to a prime ideal is regular local and has its Krull dimension $=$ its global dimension $\leqslant$ gl.dim $R<\infty$. For sufficiency, by [2], $R$ is either a $(0,1,2)$-ring, or an $(n, n, 2)$-ring, with $n \neq 0$. For the latter case, every $R_{p}$, where $p$ is a prime ideal of $R$, has gl.dim $R_{p}=$ Krull dimension $R_{p} \leqslant$ Krull 
dimension $R<\infty$. By $2 . \hat{7}$ and 2.8 , since f.p.dim $R=2>\sup \left\{\right.$ f.p.dim $R_{m} \mid m$ is a maximal ideal of $R$ \}, and w.gl.dim $R=\operatorname{gl} . \operatorname{dim} R$, we have

$$
\begin{aligned}
\operatorname{gl} \cdot \operatorname{dim} R & =\sup \left\{\operatorname{gl} \cdot \operatorname{dim} R_{m} \mid m \text { is a maximal ideal of } R\right\} \\
& =\sup \left\{\operatorname{gl} \cdot \operatorname{dim} R_{p} \mid p \text { is a prime ideal of } R\right\}
\end{aligned}
$$

This shows that gl.dim $R<\infty$.

In fact, the above result is also true for any coherent $(n, n, m)$-ring that is locally Noetherian.

The following result is obvious.

Proposition 2.10. $\mathscr{C}$ is closed under localization.

Proposition 2.11. If $R \in \mathscr{C}$, and w.gl.dim $R<\infty$, then $R\left[X_{1}, X_{2}, \ldots, X_{n}\right]$ is coherent, for any positive integer $n$.

Proof. By [1], it suffices to show that $R_{p}\left[X_{1}, X_{2}, \ldots, X_{n}\right]$ is coherent, for all prime ideals $p$ of $R$. This follows from 2.7 and the Hilbert Basis Theorem.

3. Rings of global dimension 2. In [3], it was shown that quasi-local rings of global dimension 2 are of three types: Noetherian rings, valuation rings and umbrella rings.

Since a quasi-local ring of global dimension 2 must be a coherent domain, and a domain with weak global dimension 0 must be a field, we conclude that a quasi-local ring of global dimension 2 must be a $(1,2,3)-$, a $(2,2,0)$-, or a $(2,2,3)$-ring. It is clear that a $(1,2,3)$-ring is a valuation ring, and that a $(2,2,0)$-ring is a Noetherian ring. Hence a $(2,2,3)$-ring must correspond to an umbrella ring.

Now let $R$ be a coherent domain with global dimension 2 . Then it must be a $(1,2,3)$-, a $(2,2,0)$-, a $(2,2,2)$ - or a $(2,2,3)$-ring. We look at each of these rings.

If $R$ is a $(1,2,3)$-ring, then it is a Prüfer domain. We divide into two cases. In case one, gl.dim $R_{p} \leqslant 1$, for all prime ideals $p$ of $R$. Then each $R_{p}$ is a $(1,1,0)$-ring, i.e. a discrete valuation ring, for any prime ideal $p \neq(0)$. Consequently, the Krull dimension of such a ring must be 1 . In case two, there is a prime ideal $p$ such that gl.dim $R_{p}=2$. Such an $R_{p}$ must be a $(1,2,3)$-ring, and so cannot be a Noetherian ring, and hence not a discrete valuation ring.

A $(2,2,0)$-ring is a Noetherian ring of Krull dimension 2. The spectrum is made up of three levels, the height 2 primes, the height 1 primes and the zero ideal.

A $(2,2,2)$-ring is locally Noetherian. This shows that the Krull dimension of $R$ is 2. If $p$ has height 2 , then $p R_{p}$ contains at least two noncomparable prime ideals of $R_{p}$. Thus $p$ contains at least two noncomparable prime ideals of $R$. By the $\Delta$-Theorem in [3], $p$ is finitely generated. By Cohen's theorem, there must be a prime ideal that is nonfinitely generated. Consequently, such a prime ideal must have height 1.

The spectrum of such a ring looks like that of a $(2,2,0)$-ring, the difference being that some prime ideals in the middle level are nonfinitely generated. 
The picture is more complicated for a $(2,2,3)$-ring. Its localization with respect to a maximal ideal (or a nonzero prime ideal) is a $(1,1,0)-$, a $(2,2,0)-$, a $(1,2,3)-$, or a $(2,2,3)$-ring, i.e. a Noetherian ring of Krull dimension 1 or 2, a valuation ring of global dimension 2, or an umbrella ring. Hence its spectrum looks like the spectra of these rings laid side by side.

\section{REFERENCES}

1. B. Alfonsi, Grade non-noethérien, Comm. Algebra 9 (1981), 811-840.

2. H. K. Ng, Finitely presented dimension of commutative rings and modules, Pacific J. Math. (to appear).

3. W. V. Vasconcelos, The rings of dimension two, Dekker, New York, 1976.

Department of Mathematics, Albion College, Albion, Michigan 49224 